\title{
Ein Beitrag digitaler Technologien im Kontext der Fahrzeugnutzung zur (ökologischen) Nachhaltigkeit
}

\author{
Alexander Stocker (iD
}

Eingegangen: 13. August 2020 / Angenommen: 16. Oktober 2020 / Online publiziert: 5. November 2020 (C) Springer Fachmedien Wiesbaden GmbH, ein Teil von Springer Nature 2020

Zusammenfassung Schon seit Jahren spielen die Themen Nachhaltigkeit und Digitalisierung in fast allen Lebensbereichen eine bedeutende Rolle. Zu diesen Bereichen zählen auch Mobilität als Beweglichkeit von Personen und Gütern im geographischen Raum sowie Transport als der Prozess der Bewegung von Personen und Gütern von einem Ort zum anderen. Dabei übt Digitalisierung einen transformativen Einfluss auf Mobilität und Transport aus, und es stellt sich die Frage, wieviel Digitalisierung zur Nachhaltigkeit beitragen kann. Menschen können bereits heute über ihr Mobiltelefon verschiedene Mobilitätsangebote buchen und mit digitalen Assistenzsystemen zur effizienten, sicheren und sparsamen Fahrzeugnutzung angeleitet werden. Durch die zunehmende Automatisierung der Fahrfunktionen sollen Sicherheit, Komfort und Effizienz noch weiter verbessert werden. Der vorliegende Beitrag diskutiert anhand dreier Fallbeispiele, datenbasierte Dienste und mobile Anwendungen für Fahrer, Fahrerassistenzsysteme für „Eco-Driving“ sowie nachhaltige Mobilitätsdienstleistungen und Plattformen, wie der Einsatz digitaler Technologien im Kontext der Fahrzeugnutzung einen Beitrag zur (ökologischen) Nachhaltigkeit liefern kann. Der Beitrag schließt mit einem Ausblick auf zu erwartende Entwicklungen rund um die Themen datenbasierte Services, digitale Plattformen und Fahrzeugautomatisierung.

Schlüsselwörter Mobilität · Transport · Informationssysteme · Assistenzsysteme · Automatisiertes Fahren

\footnotetext{
A. Stocker $(\bowtie)$

Virtual Vehicle Research GmbH, Inffeldgasse 21a, 8010 Graz, Österreich

E-Mail: alexander.stocker@v2c2.at
} 


\title{
A contribution of digital technologies in the context of vehicle use to (ecological) sustainability
}

\begin{abstract}
For years, sustainability and digitization have played a significant role in almost all areas of life. These include mobility as the movement of people and goods in geographical space, and traffic as the process of transporting people and goods from one place to another. Digitization exerts a transformative influence on mobility and transport, and the question arises as to how far digitization can contribute to sustainability. Already today, people can book various mobility offers via their cell phones and be guided to efficient, safe, and economical use of vehicles with digital assistance systems. The increasing automation of driving functions is expected to further improve safety, comfort, and efficiency. Using three case studies, data-based services and mobile applications for drivers, driver assistance systems for eco-driving, and services and digital platforms for sustainable mobility, this article discusses how the use of digital technologies in the context of vehicle use can contribute to (environmental) sustainability. The article concludes with an outlook on expected developments in the areas of data-based services, digital platforms, and vehicle automation.
\end{abstract}

Keywords Mobility · Transport · Information Systems · Assistance Systems · Automated driving

\section{Nachhaltigkeit und Digitale Technologien}

Das klassische Nachhaltigkeitskonzept basiert auf einer langfristigen, ökologisch verträglichen Nutzung kritischer Naturkapitalbestände und ist erst in den letzten Jahren zu einem Leitprinzip der internationalen Umweltpolitik geworden (Ott und Döring 2011). Einen wichtigen Meilenstein in der politischen Verankerung des Nachhaltigkeitskonzepts stellt dabei die Konferenz der Vereinten Nationen über Umwelt und Entwicklung (UNCED Konferenz) von Rio de Janeiro im Jahr 1992 dar, welche den Menschen in den Mittelpunkt der Bemühungen um eine nachhaltige Entwicklung gerückt und ihm das Recht auf ein gesundes und produktives Leben im Einklang mit der Natur zugesprochen hat (Eissen et al. 2002). Mit dieser Positionierung des Menschen im Zentrum der Nachhaltigkeitsbestrebungen soll auch den Entwicklungs- und Umweltschutzbedürfnissen zukünftiger Generationen in einer gerechten Weise entsprochen werden.

Doch Nachhaltigkeit muss keineswegs nur aus einer ökologischen Perspektive verstanden werden, denn aktuelle Konzepte sehen den Nachhaltigkeitsbegriff durchaus breiter: Nach dem Drei-Säulenmodell der Nachhaltigkeit setzt Nachhaltigkeit als ganzheitliches Zukunftskonzept neben der ökologischen Komponente auf zwei weiteren Komponenten, die ökonomische sowie die soziale Nachhaltigkeit, welche gleichrangig berücksichtigt werden müssen (Brand und Jochum 2000). So argumentieren Elkington und Rowlands (1999), dass soziale Gerechtigkeit, wirtschaftlicher Wohlstand und Umweltqualität - die drei Schlüsselelemente der ,Triple Bottom Line“ - die Maßstäbe sein werden, an denen sich Unternehmensleistung messen 
lassen muss: Die Gesellschaft ist von der Wirtschaft abhängig, und die Wirtschaft ist wiederum vom globalen Ökosystem abhängig, dessen Gesundheit die ultimative „Bottom Line“ darstellt (Elkington 1997).

Die Vereinten Nationen (2015) haben schließlich siebzehn nachhaltige Entwicklungsziele (,Sustainable Development Goals“) definiert, welche die globalen Herausforderungen einschließlich solcher, die mit Armut, Ungleichheit, Klimawandel, Umweltzerstörung, Frieden und Gerechtigkeit zusammenhängen, thematisieren, um eine bessere und damit nachhaltigere Zukunft für alle zu erreichen. Auch die Rolle von Mobilität und Transport wird im UN Report thematisiert: So wurde im Hinblick auf die Erreichung des Ziels, Städte und menschliche Siedlungen inklusiv, sicher, widerstandsfähig und nachhaltig zu gestalten, auch empfohlen, den Zugang zu sicheren, erschwinglichen, zugänglichen und nachhaltigen Verkehrssystemen für alle $\mathrm{zu}$ ermöglichen und die Verkehrssicherheit zu verbessern, insbesondere durch den Ausbau des öffentlichen Verkehrs, unter besonderer Berücksichtigung der Bedürfnisse un- bzw. wenig geschützter Verkehrsteilnehmer (,vulnerable road users“), von Frauen, Kindern, Menschen mit Behinderungen und älteren Menschen. Die mögliche Rolle der Digitalisierungswelle als Befähiger zur Erreichung dieser Nachhaltigkeitsziele werden in diesem Report weitestgehend ausgeklammert.

In ihrem Report „Digital Revolution and Sustainable Development“ diskutieren Messner et al. (2019), wie sich digitale Technologien gezielt dazu einsetzen lassen, um die Welt im Hinblick auf die Erreichung der von den Vereinten Nationen definierten siebzehn Entwicklungsziele nachhaltiger zu gestalten. Auch zu Mobilität und Transport finden sich entsprechende Beiträge: Beispielsweise werden Elektro- und Plug-in-Hybridfahrzeuge als eine große Hoffnung beschrieben, um die schädlichen Umweltauswirkungen von Fahrzeugen zu reduzieren, unter der Voraussetzung, dass der benötigte Strom nachhaltig, ohne Luftverschmutzung und Treibhausgasemissionen, produziert wird. Auch die fortschreitende Automatisierung von Fahrzeugen sowie damit möglich werdende Effekte, wie beispielsweise die Reduktion der Fahrzeugflotte und des Ressourcenverbrauchs bei „Shared Ownership“, einer geteilten Nutzung von Vermögenswerten, wird in einem Gedankenexperiment thematisiert. Die geteilte Nutzung von Fahrzeugen anstatt des klassischen Eigentums von Fahrzeugen kann den Ressourcenverbrauch nachhaltig vermindern und einen positiven Beitrag zur Ressourceneffizienz leisten.

\section{Nachhaltigkeit in der Wirtschaftsinformatik}

Digitalisierung, digitale Technologien, digitale Innovation und Digitale Transformation sind aus der öffentlichen Diskussion nicht mehr wegzudenken. Es scheint, als habe „Digital“ etablierte Begriffe, wie Informationstechnologien (IT) und Informations- und Kommunikationstechnologien (IKT) zumindest im öffentlichen Diskurs weitgehend ersetzt. Letztendlich wird der Einsatz bekannter Technologien, wie Maschinelles Lernen, Künstliche Intelligenz und Internet der Dinge unter dem Deckmantel der „Digitalisierung“ geführt.

In der wissenschaftlichen Literatur finden sich zahlreiche Bestrebungen, auf Synergien zwischen Informationssystemen (IS), Informationstechnologie (IT) und dem 
Erzielen von Nachhaltigkeitseffekten hinzuweisen: So wurde etwa Green IS als eine Subdisziplin von Information Systems (IS) etabliert um aufzuzeigen, dass gerade die IS Community einen signifikanten Beitrag zur Reduzierung von Treibhausgasemissionen und zur Eindämmung der Auswirkungen des globalen Klimawandels sowie anderer Umweltprobleme leisten kann (vom Brocke et al. 2013). Diskussionen über nachhaltige Informationstechnologie (Green IT) beziehen sich auf Aspekte wie beispielweise Ressourcenreduktion im Produktlebenszyklus, Reduktion von Energie in der Nutzung von IT sowie, Reduktion von Energie in anderen Sektoren durch die Nutzung von IT (Kosch und Wagner 2010). Das Bekenntnis zu nachhaltiger Informationstechnologie gilt als eine Managementaufgabe. Daher werden die Wahrnehmung der Entscheidungsträger in Bezug auf Green IS und die Folgen der Einführung von Green IS ebenfalls thematisiert (Gholami et al. 2013). Informationssysteme können einen wichtigen Beitrag zur ökologischen Nachhaltigkeit leisten, beispielsweise durch Gestaltung einer positiven Meinungsbildung über Umwelt, durch Transformation zu nachhaltigen Geschäftsprozessen und Arbeitspraktiken in Organisationen, oder durch die Verbesserung der ökologischen und wirtschaftlichen Leistungsfähigkeit eines Unternehmens (Melville 2010).

Dennoch argumentieren Autoren, dass die Wirtschaftsinformatik das Problem der Nachhaltigkeit nur langsam erkannt hat und sich äußerst überschaubar an der Entwicklung ökologisch nachhaltiger Geschäftspraktiken beteiligt (Watson et al. 2010). Nur langsam wächst das Verständnis, dass Informationssysteme (IS) zur Gestaltung und Umsetzung nachhaltiger Prozesse, Dienstleistungen und Produkte einen signifikanten Beitrag leisten können (vom Brocke et al. 2013).

Mehr Beachtung findet der Nachhaltigkeitsbegriff im Supply Chain Management, etwa im Bilden einer nachhaltigen Wertschöpfungskette (Kumar et al. 2012). Die Nutzung von Informationssystemen kann einen wesentlichen Beitrag zur Unterstützung eines nachhaltigen Supply Chain Managements leisten (de Camargo and Jabbour 2017). Auch der Zusammenhang zwischen Nachhaltigkeit und Kreislaufwirtschaft wird immer wieder thematisiert. Unter Kreislaufwirtschaft wird ein regeneratives System verstanden, in dem Ressourceneinsatz, Abfall, Emissionen und Energieverluste durch Verlangsamung, Schließung und Verengung von Material- und Energiekreisläufen minimiert werden, was durch langlebige Konstruktion, Wartung, Reparatur, Wiederverwendung, Wiederaufarbeitung und Recycling erreicht werden kann (Geissdoerfer et al. 2017). Der gezielte Einsatz von Informationstechnologie kann einen wesentlichen Beitrag zur Umsetzung einer solchen Kreislaufwirtschaft leisten (Pagoropoulos et al. 2017). Laut dem Umweltbundesamt ${ }^{1}$, Deutschlands zentraler Umweltbehörde, lagen die Altfahrzeugverwertungsquote in Deutschland für 2018 bei 95,7 Gewichtsprozent und die Recyclingquote (inklusive Wiederverwendung von Ersatzteilen) bei 87,1 Gewichtsprozent.

Auch zu nachhaltiger Mobilität und nachhaltigem Transport kann die IS Community einen wesentlichen Beitrag leisten, da auch dort der Einsatz von Informationssystemen eine wesentliche Rolle spielt (Kaiser et al. 2018). Das kann beispielweise

\footnotetext{
1 Umweltbundesamt, Altfahrzeugverwertung und Fahrzeugverbleib: www.umweltbundesamt.de/daten/ ressourcen-abfall/verwertung-entsorgung-ausgewaehlter-abfallarten/altfahrzeugverwertungfahrzeugverbleib.
} 
im Design und in der Evaluierung von Informationssystemen rund um das Thema der Fahrzeugnutzung, im Design von Geschäftsmodellen für digitale Mobilitätslösungen, oder in der Konzeption digitaler Mobilitätsplattformen passieren (Stocker et al. 2017).

\section{Nachhaltigkeit und Mobilität}

Die Mobilitätsforschung hat sich als die Forschung zu Bewegung und Beweglichkeit im Raum von einer ingenieurs- und wirtschaftswissenschaftlich geprägten Technikforschung mittlerweile zu einem stark interdisziplinären Forschungsfeld weiterentwickelt. Mobil zu sein ist heute für viele Menschen zu einer Selbstverständlichkeit geworden und stellt ein Grundbedürfnis dar. Vor allem die Alltagsmobilität hat stark zugenommen, wobei rund zwei Drittel aller Wege im urbanen Raum zurückgelegt werden (Herwig 2017).

Der gesamte Straßenverkehr des 21. Jahrhunderts steht vor einer enormen Herausforderung, denn es gilt, $\mathrm{CO}_{2}$-Emissionen $\mathrm{zu}$ begrenzen und den endlichen Energieträger Erdöl langfristig zu ersetzen (Bozem et al. 2013). Der Verkehrssektor verantwortet weltweit einen Anteil von rund $20 \%$ der energiebedingten Treibhausgasemissionen (Ruhrort 2019). Es steht fest, dass Mobilität nachhaltiger werden muss und es dazu die richtigen Strategien benötigt. Eine geeignete Strategie zur Erreichung einer nachhaltigen Mobilität von Personen und Gütern mit Bezug zur Thematik Verkehr umfasst nach Ansicht des Umweltbundesamts ${ }^{2}$ die vier Felder, Verkehr vermeiden, Verkehr auf umweltverträglichere Verkehrsträger verlagern, Energieeffizienz erhöhen sowie, postfossile, treibhausgasneutrale Kraftstoffe und Strom nutzen.

Als Fortbewegungsmittel spielen Kraftfahrzeuge heute eine treibende Rolle. Laut eines Berichts des Bundesministeriums für Verkehr und Infrastruktur ${ }^{3}$ bleibt der PKW im deutschsprachigen Raum vor allem außerhalb der Städte das beliebteste Verkehrsmittel, und in Deutschland kommen auf 1000 Einwohner 527 Personenkraftfahrzeuge in Privathaushalten. Doch im Bereich der Verkehrsmittel wird heute bereits viel über Veränderungen im Hinblick auf alternative Antriebe und Kraftstoffe über Elektromobilität bis hin zu Automatisiertem Fahren und einer möglichen Neugestaltung des Verkehrs und Mobilitätssystems diskutiert (Kollosche und Schwedes 2016). So können beispielsweise eine effizientere Nutzung bestehender Verkehrsinfrastrukturen und eine erhebliche Reduktion verkehrsbedingter Schadstoffemissionen dadurch erreicht werden, dass künftig hochautomatisierte Fahrzeuge mit intelligenten Verkehrssteuerungen Informationen austauschen (Clausen und Klinger 2018).

Die Wirtschaftsinformatik kann durch die Gestaltung von Informationssystemen einen wesentlichen Beitrag zu innovativen und nachhaltigen Geschäftsmodellen auf dem Gebiet der Mobilität leisten, denn vor allem die Vernetzung von Fahrzeugen,

\footnotetext{
2 Umweltbundesamt, Nachhaltige Mobilität, www.umweltbundesamt.de/themen/verkehr-laerm/ nachhaltige-mobilitaet.

${ }^{3}$ Bundesministerium für Verkehr und digitale Infrastruktur, Mobilität in Deutschland, www.bmvi.de/ SharedDocs/DE/Anlage/G/mid-ergebnisbericht.pdf, zugegriffen am 28. Juli 2020.
} 
Infrastruktur und Kunden durch Informationssysteme birgt enorme Potentiale, umweltfreundlichen Verkehr nutzerfreundlich umzusetzen (Busse et al. 2014). Auch die Nutzung von Informationssystemen in Fahrzeugen und die daraus resultierende Unterstützung der Fahrzeugnutzer bei Fahraufgaben spielt eine wesentliche Rolle, vor allem wenn diese Systeme als Assistenzsysteme zu einem sicheren und ökonomisch nachhaltigen Fahrverhalten motivieren bzw. anleiten (Kaiser et al. 2018). Digitale Plattformen sind das zentrale Element neuer Geschäftsmodelle wie beispielsweise der Sharing Economy, welche auch im Mobilitätssektor eine zunehmende Rolle spielt und langfristig zu einem Wandel im Konsumverhalten führen kann.

Im nachfolgenden Kapitel wird nun in drei Fallbeispielen gezeigt, wie digitale Technologien im Kontext der Fahrzeugnutzung einen Beitrag zur ökologischen Nachhaltigkeit leisten können.

\section{Digitale Technologien im Kontext von Fahrzeugnutzung}

\subsection{Fallbeispiel 1: Datenbasierte Dienste und Smartphone-Anwendungen für Fahrer}

Moderne Fahrzeuge sind als Hochleistungsrechner auf Rädern mit einer leistungsfähigen Sensorik ausgestattet und generieren eine Vielzahl an Daten über sich selbst und ihre Umgebung, um Funktionalität, Komfort und Sicherheit zu gewährleisten und Fahrer bei der Durchführung der Fahraufgabe bestmöglich zu unterstützen (Stocker et al. 2017). Die von dieser Sensorik gesammelten Daten ermöglichen heute eine breite Palette innovativer Anwendungen, auch im Kontext einer nachhaltigen Mobilität.

Die Möglichkeit der Entwicklungen datengetriebener bzw. datenbasierter Services hat mittlerweile zu einer unüberschaubaren Flut von Startups geführt, welche unterschiedliche mobile Anwendungen für Fahrende bereitstellen. Diese reichen von einer bloßen Anzeige von Statistiken zur aktuellen Fahrt, ähnlich zu Quantified Self-Anwendungen, über die Selbstdiagnose von Fehlern für Fahrzeugnutzer, bis hin zur Verbrauchsoptimierung während der Fahrt und dem Vergleich von Fahrstil und Fahreffizienz mit Personen im eigenen sozialen Netzwerk.

Mittlerweise setzen in diesem Umfeld tätige Startups wie beispielsweise Zendrive.com, Vin.li, Moj.io oder Dash.by ganz auf die Etablierung digitaler Ökosysteme. Sie stellen die digitalen Plattformen zur Verfügung und bieten damit anderen Akteuren die Möglichkeit, eigene datenbasierte Services durch Nutzung ihrer Infrastruktur zu generieren. Dabei kann die Detektion von Fahrstil, Fahrpraktiken und Fahrmuster und dem daraus errechenbaren Fahrrisiko dazu beitragen, dass Fahrende ihr Fahrverhalten verändern, um die Sicherheit im Straßenverkehr für alle zu erhöhen (Kaiser et al. 2020). Letztendlich korreliert eine sichere, sanfte und defensive Fahrweise zumeist mit einer ökonomisch freundlichen Fahrweise und reduziert Ressourcenverbrauch und Emissionen. Mit ihren datenbasierten Diensten treten diese Startups in Konkurrenz zu den klassischen Fahrzeugherstellern.

Beispielsweise bieten Pace und T-Mobile bzw. Magenta Car Connect mobile Anwendungen, mit denen Fahrende auf ihrem Mobiltelefon relevante durch die Fahr- 

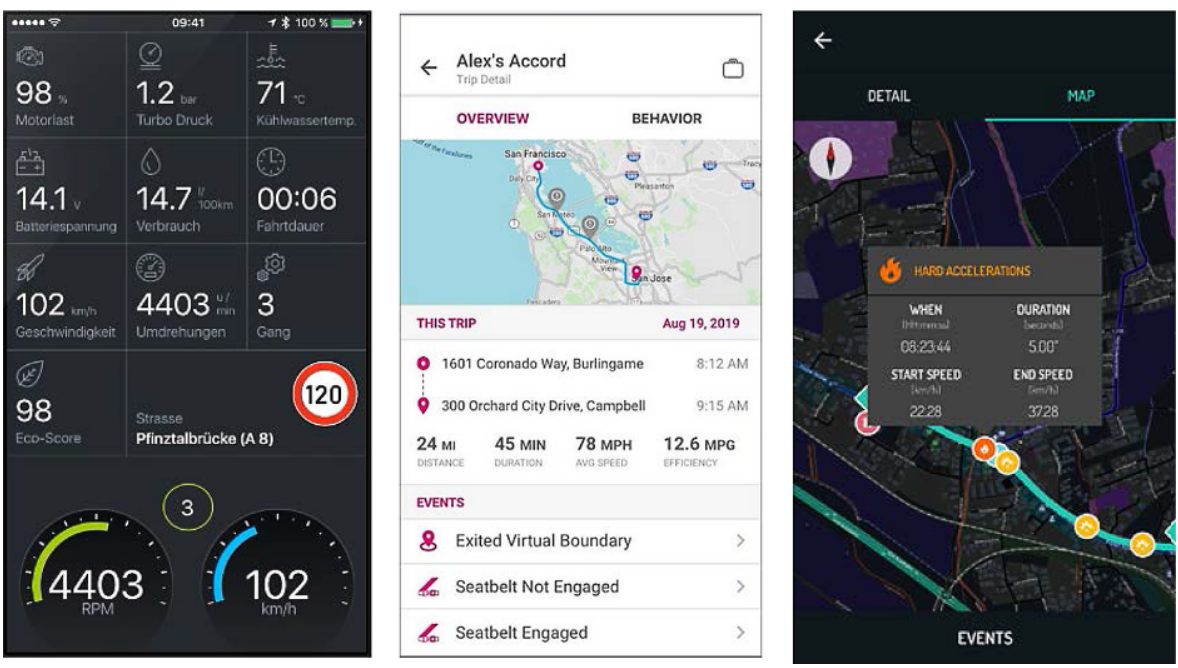

Abb. 1 Pace Performance Monitor (Pace.car, https://www.pace.car/en/features/performance-monitor), Car Connect (telekom.de), SCOTT Driving App (Kaiser et al. 2020)

zeugsensoren gesammelte Daten wie beispielsweise Geschwindigkeit, Verbrauch, Motorlast oder Motordrehzahl abrufen und anschaulich in einer grafischen Oberfläche visualisieren können (Abb. 1). Letzteres bedient sich dabei der Technologie von Moj.io. Dazu verbinden Fahrzeugnutzer ihr Fahrzeug mit ihrem Mobiltelefon und einem an die On Board Diagnostic (OBD) Schnittstelle ihres Fahrzeugs gesteckten Bluetooth-Dongle. Durch eine Kombination von über diese OBD Schnittstelle abgerufenen Fahrzeugdaten lassen sich für Fahrer interessante Statistiken - und in einer Kombination mit den Daten aus den Sensoren des Mobiltelefons - sogar Streckenvisualisierungen und streckenabhängige Fahrprofile erstellen. Auch kann etwa der Verbrauch des Fahrzeugs aus den Fahrzeugdaten berechnet und in einer Übersichtstatistik neben Distanz, Dauer und Geschwindigkeit zu den gefahrenen Fahrstrecken angezeigt werden. Damit ist beispielsweise auch die Erstellung individueller oder streckenabhängiger Effizienzprofile möglich. Sollten Fahrzeugnutzer in der mobilen Anwendung auch Fahrzeugpreise, Treibstoffpreise oder Wartungskosten erfassen, können die Kosten einer Fahrt angezeigt und erklärt werden. Auch die Detektion fahrstilprägender Ereignisse, wie beispielsweise starkes Bremsen, starkes Beschleunigen, schnelles Kurvenfahren oder generelle Geschwindigkeitsübertretungen können Fahrzeugnutzern angezeigt werden (Kaiser et al. 2020). Gerade aus solchen Ereignissen können je nach Fahrweise Sicherheits- bzw. Ökologiepunkte abgeleitet werden, um einen Vergleich mit anderen Fahrenden hinsichtlich der Maximierung der eigenen Sicherheit im Straßenverkehr und der Minimierung des Ressourcenverbrauchs zu ermöglichen und damit zu einem sicheren, ökonomisch und ökologisch nachhaltigen Fahrverhalten anzuregen.

Nicht alle angebotenen datengetriebenen Dienste sowie die dahinterstehenden Geschäftsmodelle konnten sich ökonomisch als nachhaltig erweisen, und eine Reihe an Startups musste ihren Betrieb bereits einstellen. Beispielsweise musste laut 
Techcrunch ${ }^{4}$ das im Jahr 2011 gegründete Startup Automatic.com trotz des Einstiegs des amerikanischen Satellitenradio-Anbieters SiriusXM um rund 100Mio. USD die Geschäftstätigkeit am 28 Mai 2020 gemäß eines Berichts des Magazins Cnet $^{5}$ beenden.

$\mathrm{Zu}$ den Investoren in solche Startups gehören neben dem Aftermarket-Sektor auch Versicherungsunternehmen, welche an Technologien zur Fahrstilerkennung und Fahrerprofilbildung interessiert sind. Im Hinblick auf die Effektivität fahrstilabhängiger Versicherungsverträge lassen sich bereits interessante Studienergebnisse aus der Literatur identifizieren: Der Einsatz fahrstilabhängiger Versicherungen wie ,,pay-asyou-drive“ oder ,pay-how-you-drive“ führt beispielsweise dazu, dass vor allem junge Fahrer weniger Geschwindigkeitsübertretungen begehen (Bolderdijk et al. 2011). Die nachhaltige Verbesserung von Fahrstil und Fahrverhalten wie beispielsweise eine Verringerung starker Brems- und Beschleunigungsvorgänge, eine Verringerung der jährlichen Fahrleistung bzw. die Anpassung der Tageszeit zu der gefahren wird, wird bei solchen Versicherungsverträgen aktiv durch das Setzen eines finanziellen Anreizes erreicht. Damit können solche Verträge auch für die Erreichung von Nachhaltigkeitszielen von Nutzen sein, etwa für die Reduktion von Verkehrsstaus und Schadstoffemissionen.

\subsection{Fallbeispiel 2: Im Fahrzeug verbaute Fahrerassistenzsysteme für ,Eco- Driving6"}

Die Fahrzeugindustrie investiert bereits seit einigen Jahren enorme Summen in die Automatisierung von Fahrzeugen. Durch automatisiertes und vernetztes Fahren sollen Unfallzahlen weiter gesenkt, Verkehrssicherheit drastisch erhöht und auch die Umweltbelastung verringert werden. Dennoch erfolgt diese Automatisierung als schrittweiser Prozess und führt zunächst über den verstärkten Einsatz innovativer Fahrerassistenzsysteme in Fahrzeugen, welche dazu in der Lage sind, Fahrenden mit der Zeit immer mehr Fahraufgaben abzunehmen, um Sicherheit, Komfort und Effizienz zu verbessern, von diesen aber auch aktiv eingesetzt werden müssen. Dabei steigt das Vertrauen in solche Systeme in der Regel mit der Nutzung (Neuhuber et al. 2020).

Im Hinblick auf die Verbesserung der ökologischen Nachhaltigkeit sind vor allem jene Typen von Fahrerassistenzsystemen relevant, welche den Fahrstil einer Person in dieser Hinsicht positiv beeinflussen können. Solche häufig unter dem Begriff „Eco-Driving“ zusammengefasste Systeme sind dazu in der Lage, Treibstoffverbrauch und $\mathrm{CO} 2$ Ausstoß zu reduzieren, wenn sie entsprechend genutzt werden (Barkenbus 2010). Eco-driving umfasst als Fahrstrategie Fahraufgaben wie sanfte Beschleunigung, frühes Schalten in einen höheren Gang, sowie eine proaktive Beachtung von Verkehrsfluss und Straßenführung, um plötzliches Bremsen und nachfolgendes starkes Beschleunigen zu vermeiden (McIlroy und Stanton 2015). Assis-

\footnotetext{
4 Techcrunch, SiriusXM acquires connected car company Automatic for over $\$ 100 \mathrm{M}$, https://techcrunch. com/2017/04/27/siriusxm-acquires-connected-car-company-automatic-for-over-100m.

5 Cnet, Automatic connected car service ends May 28, https://www.cnet.com/roadshow/news/automaticconnected-car-service-dead-may-coronavirus.
} 
tenzsysteme für Eco-Driving geben Fahrenden allgemeine Ratschläge wie langsames beschleunigen, Geschwindigkeit reduzieren, oder in den richtigen Gang zu schalten.

Auch in diesem Kontext wurden bereits zahlreiche wissenschaftliche Studien durchgeführt. Barkenbus (2010) hat beispielsweise in einer Studie gezeigt, dass ein mit solchen Assistenzsystemen motiviertes ökologisches Fahren den Kraftstoffverbrauch im Durchschnitt sogar um 10\% senkt. Eine weitere Studie belegt ebenfalls den positiven Effekt solcher Fahrerassistenzsysteme: Die Bereitstellung von Ratschlägen zum kraftstoffeffizienten Fahren in Echtzeit kann eine Verbesserung der Kraftstoffeffizienz um 4,1\% bewirken und das sogar ohne Erhöhung der Fahrdauer und ohne Verringerung der Durchschnittsgeschwindigkeit. Effizienzeinsparungen werden vor allem durch die Beachtung einer geeigneten Schaltstrategie ermöglicht: Es gilt, den Einsatz niedriger Gänge durch eine vorausschauende Planung zur Vermeidung unnötiger Stopps zu minimieren und die Verwendung des höchsten Ganges, wenn immer möglich und vom System empfohlen, zu maximieren (Birrel et al. 2014). Auch eine Studie zu den Einsparungspotenzialen beim Einsatz von Smartphone-basierten Assistenzsystemen in einem Elektrofahrzeug kommt zu einem positiven Ergebnis. So konnte der Energieverbrauch eines Elektrofahrzeugs in der Gruppe der Assistenzsystemnutzer im Vergleich zur Kontrollgruppe um 9,5\% verringert werden (Degirmenci et al. 2015). Diese Einsparungen sind ein Resultat der Veränderung menschlichen Verhaltens während der Fahrt, welche durch das Assistenzsystem vorgeschlagen wurde.

Ansätze zur Stimulierung von Verhaltensänderungen im Hinblick auf ein nachhaltigeres Konsumverhalten werden heute häufig unter dem Begriff „Nudging“ zusammengefasst (Lehner et al. 2016). Nudging wird dabei als die Verwendung von Design-Elementen für Benutzeroberflächen verstanden, um damit das Verhalten von Menschen in Umgebungen zu steuern, in welchen einem Nutzer unterschiedliche Möglichkeiten zur Verfügung stehen (Weinmann et al. 2016). Ein modernes Fahrzeug, vielfältige Fahraufgaben sowie die mittlerweile äußerst komplex gewordene Benutzeroberfläche dieses Fahrzeugs stellen eine solche Umgebung dar.

Auch durch „Gamification“ soll ein bestimmtes Benutzerverhalten stimuliert werden. Unter Gamification wird die Anwendung von Design-Elementen aus der Spieleindustrie und die Nutzung von Spielprinzipien in Nicht-Spielkontexten verstanden (Hamari et al. 2014). Geeignete Spieldesignelemente sind beispielsweise die Dokumentation von Nutzerverhalten, Einsatz von Punktesystemen, Badges und Trophäen, sowie die Verwendung von Ranglisten, Levels, Gruppenaufgaben, Herausforderungen und Avatare (Blohm und Leimeister 2013). Die Benutzeroberfläche eines Fahrzeugs stellt einen geeigneten Kontext für den Einsatz von Gamification dar. Das Design datengetriebener Services im Kontext der Fahrzeugnutzung und das Design der Benutzerschnittstelle von Assistenzsystemen profitieren heute immens von Ansätzen aus der Gamification-Forschung, um Fahrer noch stärker zu einer ökonomischeren und ökologischeren Fahrweise anzuregen (Magana und Munoz-Organero 2015).

Der Großteil der Fahrzeughersteller ermöglicht Fahrzeugnutzern die Wahl einer Öko-driving Strategie und stellt dazu beispielsweise Spontan- und Durchschnittsverbrauch numerisch bzw. sogar grafisch dar. Fahrzeughersteller nutzen auch Elemente aus dem Bereich der Gamification, wie beispielsweise sich ändernde Farben durch 

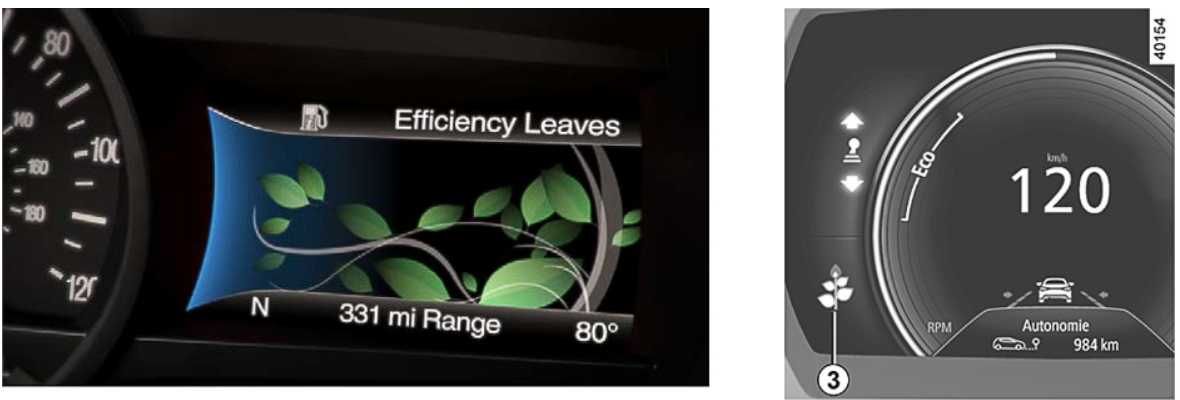

Abb. 2 Ford Dashboard mit EcoGuide (Quelle: Ford EcoGuide, https://owner.ford.com/support/howtos/vehicle-features/dashboard-instrument-cluster/smartgauge-with-ecoguide.html) und Renault Driving Style Indicator (Quelle: Renault driving advice, eco-driving: https://gb.e-guide.renault.com/eng/Kadjar/ DRIVING-ADVICE-ECO-DRIVING)

den gezielten Einsatz von LED-Elementen im Fahrzeuginnenraum sowie von grafischen Symbolen im Dashboard, welche sich je nach Fahrstrategie und Beschleunigungsverhalten dynamisch anpassen. Das soll Fahrer dazu motivieren, ihren Fahrstil wieder an eine ökonomischere Fahrweise anzupassen. Beispielsweise verwenden Ford und Renault gezielt das Symbol „Blatt“, um Fahrer zu einem sparsameren Fahrstil anzuregen. Je mehr Blütenblätter im Dashboard des Fahrzeugs angezeigt werden, desto ruhiger und sparsamer ist ein Fahrer unterwegs. Verschwindende bzw. sogar verwelkende Blätter sollen den Fahrer wieder zu einer ökologisch freundlicheren Fahrweise anregen, grüne Blätter bestätigen dem Fahrer eine solche Fahrweise (Abb. 2).

Aufgrund der begrenzten Reichweite ist gerade bei Elektrofahrzeugen ein ruhiger und sanfter Fahrstil unabdingbar. Beispielsweise zeigt der Elektrofahrzeughersteller Tesla neben der Entwicklung des Energieverbrauchs in Echtzeit grafisch an, wieviel Energie durch Rekuperieren wieder in das System zurückgeführt wird. Beim Rekuperieren wird die Bewegungsenergie eines Fahrzeugs wieder in elektrische Energie zurück umgewandelt, um so die Reichweite von Elektrofahrzeugen zu steigern. Das

Abb. 3 Tesla Energy Graph (Quelle: Tesla Energy Graph: https://www.tesla.com/sites/ default/files/model_s_owners_ manual_north_america_en_us. pdf)

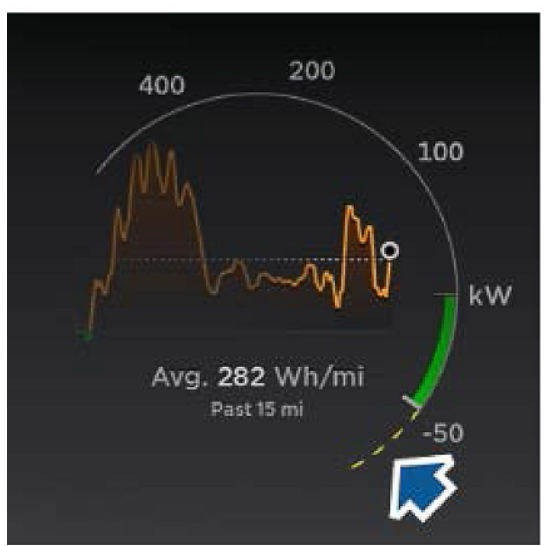


Fahrzeug rekuperiert immer dann, wenn der Fahrer den Fuß vom Fahrpedal nimmt. Mehr Rekuperation führt zu vergrößerter Bremswirkung bei gleichzeitig größerer Rückgewinnung von Energie. Je höher die Fahrgeschwindigkeit, desto mehr Energie wird aufgrund des exponentiell steigenden Luftwiderstands des Fahrzeugs verbraucht. Der in Abb. 3 dargestellte Energy Graph von Tesla will Informationen wie den Energieverbrauch in Wattstunden je Meile über die letzten 15 Meilen anschaulich zugängig machen und Fahrzeugnutzer so zu einem ressourcenfreundlicheren Fahren motivieren, um die Reichweite des Elektrofahrzeugs zu optimieren. Geht die Kurve im Graph nach oben, wurde mehr Energie benötigt, geht die Kurve dagegen nach unten, wird weniger Energie benötigt. Ein ressourcenschonender Fahrstil vermeidet, dass die Kurve zu hoch nach oben ausschlägt.

\subsection{Fallbeispiel 3: Nachhaltige Mobilitätsdienstleistungen \& Plattformen}

In der Regel stehen Reisenden heute eine Reihe von Verkehrs- bzw. Transportmittel zur Verfügung, um von ihrem Ausgangsort zum gewünschten Zielort zu gelangen. Dazu zählt immer noch der Personenkraftwagen. Während in den ersten beiden Fallbeispielen Fahrer eines Personenkraftwagens durch fahrzeugungebundene Systeme (Fallbeispiel 1) und fahrzeuggebundene Systeme (Fallbeispiel 2) zu einem ökologisch und ökonomisch nachhaltigeren Fahrverhalten motiviert werden sollten, steht im dritten Fallbeispiel die Nutzung nachhaltiger Mobilitätsdienste und Plattformen wie beispielsweise Car Sharing oder Ride Sharing im Mittelpunkt. Durch die gezielte Nutzung solcher Dienste lässt sich ein wesentlich größerer ökologischer Nachhaltigkeitseffekt erzielen.

Mit dem Aufkommen prominenter digitaler Plattformen wie beispielweise Uber, Lyft und Airbnb ist die Sharing Economy zu einem aufstrebenden wirtschaftlichtechnologischen Phänomen geworden. Derart angebotene Mobilitätsleistungen, sogenannte Digital Shared Mobility Services (Herrenkind et al. 2020), können die Effizienz der vorhandenen Transportmittel deutlich steigern, beispielsweise durch die Bündelung einzelner Fahrten. Der Grundgedanke hinter dem Konzept der Sharing Economy besteht darin, dass persönliche Besitztümer einer Person oft ungenutzt bleiben und daher für bestimmte Zeiträume mit anderen Menschen geteilt werden können, die sie benötigen (Zimmermann et al. 2018). Anstatt Produkte zu kaufen und zu besitzen, sind Verbraucher zunehmend daran interessiert, sie zu leasen und mit anderen zu teilen.

Das Prinzip eines gemeinschaftlichen Konsums von Gütern trifft auch immer mehr für Fahrzeuge zu, indem diese gemeinsam genutzt werden. Carsharing besteht aus einer Gruppe zahlender Personen, die periodisch auf eine Fahrzeugflotte zugreifen, um diese fast ausschließlich für kürzere, lokale Fahrten zu verwenden (Bardhi und Eckhardt 2012). Im Gegensatz dazu bezieht sich Ride Sharing auf ein Verkehrsmittel, bei dem Reisende ein Fahrzeug für eine Fahrt teilen und dabei auf Fahrtkosten wie Benzin-, Maut- und Parkgebühren mit anderen teilen, die eine ähnliche Reiseroute und einen ähnlichen Zeitplan haben (Furuhata et al. 2013). Bei Carsharing wird also das Fahrzeug selbst geteilt, während es bei Ride Sharing um die gemeinsame Nutzung einer Fahrtstrecke geht. 
Im deutschsprachigen Raum ist Carsharing kein neues Phänomen: 1988 gilt in Deutschland als das Geburtsjahr für Carsharing, und Berlin war die erste Stadt, in der ein solches Angebot mit zunächst fünf Nutzern und einem einzigen Fahrzeug geboten wurde (Loose 2014). Im Jahr 1994 gab es bereits 69 Anbieter, die meisten davon als Verein gegründet, und im Jahr 2001 begann die Deutsche Bahn bereits mit der Organisation von umfangreichen Car Sharing Angeboten (Loose 2014). Nach Ansicht des Bundesverbands CarSharing e. V. ${ }^{6}$ gilt Deutschland überhaupt als einer der Leitmärkte für Car Sharing weltweit, auf dem 165 Car Sharing-Anbieter in 677 Städten und Gemeinden für mehr als 2 Mio. Kunden rund 18.000 geteilte Fahrzeuge bereitstellen. Bei Carsharing wird zwischen stationsgebundenen und free-floating Systemen unterschieden: Während im ersten Fall Fahrzeuge festen Standorten zugewiesen sind und von den Kunden wieder zu diesen zurückgebracht werden müssen, können im zweiten Fall die Fahrzeuge nach einer Ortung durch eine mobile Anwendung in einem definierten Gebiet abgestellt werden. Sollten Fahrzeuge in Gegenden mit einer sehr niedrigen Nachfrage abgestellt werden, beispielsweise nach einer Hinfahrt ohne einer nachfolgenden Retourfahrt, überstellen Mitarbeiter des Car Sharing Betreibers diese Fahrzeuge in der Regel wieder in Gebiete mit einer höheren Nutzerfrequenz, was natürlich mit Kosten verbunden ist.

Die Untersuchung der ökologischen Nachhaltigkeitseffekte von Carsharing ist Bestandteil einiger Studien: So haben beispielsweise Firnkorn und Müller (2011) Interviews und Umfragen mit Menschen in der Region Ulm in Bezug auf ihr Mobilitätsverhalten durchgeführt und mit Hilfe des daraus resultierenden empirischen Datensatzes die zu erwartenden positiven ökologischen Auswirkungen eines Car Sharing Anbieters, car2go, prognostiziert. Die Ergebnisse deuten auf eine Verbesserung der CO2-Emissionen pro durchschnittlichem car2go-Nutzer hin, und die $\mathrm{Au}-$ toren prognostizieren eine durchschnittliche Reduzierung von 312 bis $146 \mathrm{~kg} \mathrm{CO} 2$ pro Jahr für den durchschnittlichen car2go-Nutzer. Eine in den Niederlanden durchgeführte Studie basiert ebenfalls auf eine Nutzerumfrage und kam zum Ergebnis, dass Car Sharing Nutzer $15 \%$ bis $20 \%$ weniger Fahrzeugkilometer fahren, um $30 \%$ weniger Fahrzeuge besitzen und so zwischen 240 und $390 \mathrm{~kg}$ weniger CO2 Emissionen pro Person und Jahr ausstoßen (Nijland and van Meerkerk 2017). Eine weitere Studie aus Lissabon kommt zum Ergebnis, dass die Nutzung von Hybridfahrzeugen bzw. Elektrofahrzeugen in Car Sharing Flotten sogar zu noch größeren Einsparungen in Bezug auf Energieverbrauch (35\% bzw. 47\%) und CO2 Ausstoß (35\% bzw. $65 \%$ ) führen kann (Baptista et al. 2014).

Fest steht, dass Dienste wie Car Sharing und Ride Sharing dann zu positiven ökologischen Effekten führen, wenn sie von der breiten Masse auch genutzt werden. Die Nutzung alternativer Verkehrs- und Mobilitätslösungen hängt allerdings stark von sich wandelnden verkehrspolitischen Rahmenbedingungen, wie beispielsweise Kontingentierung des Verkehrs, sowie von einer sich wandelnden Mobilitätskultur, wie beispielsweise die abnehmende Wichtigkeit des Autobesitzes, ab (Chaloupka et al. 2015). Die größten ökologischen Nachhaltigkeitseffekte lassen sich dann erzielen, wenn Mobilitätsdienstleistungen und Plattformen eine Verkehrsverlagerung

\footnotetext{
${ }^{6}$ Bundesverband CarSharing, 30 Jahre Car Sharing in Deutschland, www.carsharing.de/sites/default/files/ uploads/30jahrecarsharing_download_0.pdf.
} 
und -vermeidung induzieren können und Menschen von ihren eigenen Fahrzeugen auf geteilte Fahrzeuge (Weber et al. 2020) bzw. auf den öffentlichen Verkehr umsteigen. Carsharing kann seine Nachhaltigkeitspotenziale vor allem dann entfalten, wenn es als Element eines multimodalen Verkehrsangebots zu einer attraktiven Alternative zum eigenen Auto wird (Tils et al. 2015). Gerade die aktuelle Covid-19 Pandemie stellt viele Car Sharing Anbieter jedoch vor große Herausforderungen und beschert ihnen zum Teil sinkende Nutzerzahlen.

\section{Diskussion}

Digitale Technologien besitzen ein großes Potenzial, die ökologische Nachhaltigkeit von Mobilität und Transport zu verbessern. Dies kann beispielsweise über durch sie ermöglichte datenbasierte Dienste und mobile Anwendungen für Fahrende (Fallbeispiel 1), in Fahrzeugen fest verbaute Assistenzsysteme (Fallbeispiel 2), oder durch digitale Plattformen und neue Geschäftsmodelle (Fallbeispiel 3) erfolgen. Der größte Effekt lässt sich vermutlich aus einer Kombination aller drei Lösungen erzielen.

Nach dem Triple Bottom Line Modell (Elkington 1997) sollen Entwicklungen auf allen drei Dimensionen nachhaltig sein, der ökologischen, der sozialen und der ökonomischen. Datengetriebene Services und mobile Anwendungen wurden in der Vergangenheit vor allem durch innovative Technologiestartups forciert, welche sich Fahrzeugdaten über die OBD Schnittstelle zunutze machen. Manche Startups haben mit dem Release einer einzelnen mobilen Anwendung gestartet und dann ihr Angebot sukzessive in Richtung der Bereitstellung einer digitalen Plattform samt der Bildung eines digitalen Ökosystems ausgeweitet (Kaiser et al. 2019). Mit dieser Plattformstrategie haben Startups dann auch Dritten ermöglicht, auf einer digitalen Infrastruktur mobile Anwendungen für Fahrende anzubieten. Diese Startups wurden teilweise mit hohen Summen an Risikokapital gefördert (Stocker et al. 2017). Dennoch mussten einige Startups ihren Betrieb bereits wieder wiedereinstellen, da sich ihre Geschäftsmodelle ökonomisch als nicht nachhaltig erwiesen haben. So musste etwa der OBD-Dongle-basierte Service von Automatic.com den Betrieb mit dem 20. Mai 2020 trotz der Übernahme durch Sirius XM um rund 100 Mio. USD einstellen ${ }^{7}$. Das kann auch ein Resultat des steigenden Wettbewerbs sein.

Auch Fahrzeughersteller bieten immer mehr datenbasierte Dienste an und machen es mit dieser Strategie den Drittanbietern nicht einfacher, diesen Markt erfolgreich zu erschließen. Oftmals werden diese Angebote unter dem Deckmantel „Connected Dienste“ zusammengefasst, so beispielsweise bei BMW („Connected Drive“" Volkswagen (,We Connect" ${ }^{“ 9}$ ) oder Daimler (,Mercedes me connect" ${ }^{" 10}$ ). Deutsche Fahrzeughersteller diskutieren vielfach darüber, die OBD Schnittstelle Schritt für Schritt ,zum Schutz der Fahrzeugintegrität“ nicht mehr zum kostenlosen Abgreifen von Fahrzeugdaten während der Fahrt freizugeben (VDA 2017a), womit viele Ge-

\footnotetext{
7 www.cnet.com/roadshow/news/automatic-connected-car-service-dead-may-coronavirus/.

8 www.bmw-connecteddrive.de/app/index.html.

9 www.portal.volkswagen-we.com.

10 www.me.mercedes-benz.com.
} 


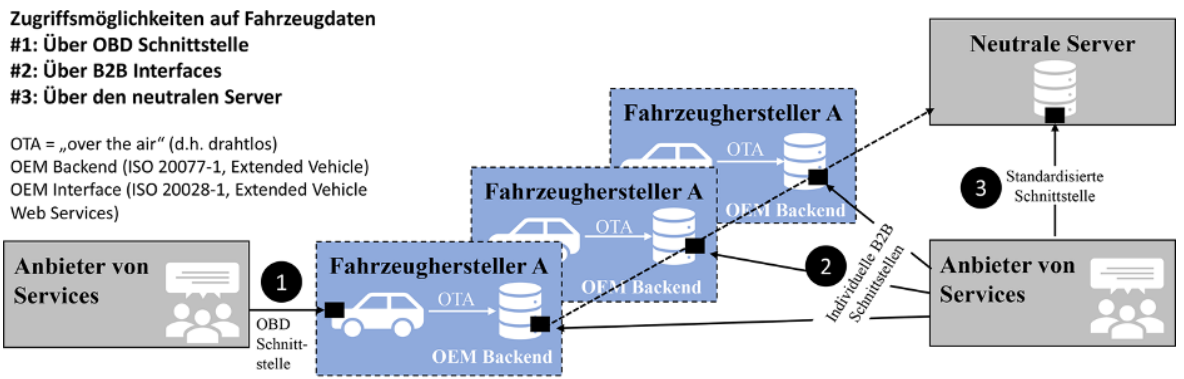

Abb. 4 Drei Möglichkeiten des Zugriffs auf Fahrzeugdaten. (Nach VDA 2017a)

schäftsmodelle besagter Drittanbieter und Startups zum Scheitern verurteilt wären. Fahrzeughersteller sollen als Administratoren die Verantwortung für einen sicheren und datenschutzkonformen Transfer von Fahrzeugdaten von einem im Fahrzeug fest installierten Gateway zu einem vom Fahrzeughersteller betriebenen Backendsystem übernehmen und die so gesammelten Daten dann über ein Business to Business Interface für Drittanwender direkt, oder über neutrale Server, zur Verfügung stellen (VDA 2017a). Ein direkter Zugriff auf Fahrzeugdaten durch Drittanbieter im Fahrzeug soll auf diese Weise aufgrund der strengen Sicherheitsvorgaben weitestgehend ausgeschlossen werden (Abb. 4).

Diesbezüglich hat die deutsche Automobilindustrie das NEVADA-Konzept entwickelt, das fahrzeuggenerierte Daten diskriminierungsfrei aus der Ferne verfügbar macht und somit ein innovatives Ökosystem an Anwendungen ermöglichen soll (VDA 2017b). In diesem Konzept bieten neutrale, nicht von Fahrzeugherstellern betriebene Server eine Möglichkeit, Fahrzeugdaten von mehreren unterschiedlichen Fahrzeugherstellern gleichzeitig im Rahmen spezieller Verträgen einzusammeln, um sie dann an Drittanbieter für Dienstleistungen weiterzuvermitteln, wodurch Serviceanbieter herstellerübergreifende Datensätze nutzen und dabei keine Einzelabstimmungen mit Fahrzeugherstellern eingehen müssen (Reich et al. 2018).

Das NEVADA-Konzept ermöglicht auch die Bildung von Datenmarktplätzen und Datenportalen wie beispielsweise Caruso-dataplace.com, auf denen Serviceanbieter Fahrzeugdaten unterschiedlicher Fahrzeughersteller in einem harmonisierten Format über eine einheitliche API beziehen können. Ein harmonisiertes Datenformat bedeutet in diesem Zusammenhang, dass Serviceanbieter die Integration ihres Dienstes mit einem solchen Datenportal über eine API nur ein einziges Mal vornehmen müssen, anstatt viele unterschiedliche Geschäftsbeziehungen mit Fahrzeugherstellern einzugehen und damit gleichzeitig mit einer Vielzahl wechselnder Datenformaten umzugehen. Es bleibt spannend, welche Daten von Fahrzeugherstellern über solche Portale überhaupt zur Verfügung gestellt werden können, zu welchem Zeitpunkt diese zur Verfügung gestellt werden, also beispielsweise während oder erst nach einer abgeschlossenen Fahrt nach erfolgtem Abstellen des Fahrzeugs, und, falls diese Daten vom Fahrzeug gestreamt werden, in welcher Frequenz. Das Angebot an unterschiedlichen Daten und die Frequenz der gestreamten Datenpunkte bestimmt die Gesamtmenge an möglichen datenbasierten Diensten. Den bereits im Artikel angesprochenen Technologiestartups bleibt somit der Weg über solche Datenportale 
offen, falls die OBD Schnittstelle von den Fahrzeugherstellern während der Fahrt geschlossen wird. Datenportale wie caruso-dataplace.com ermöglichen den Zugriff auf eine viel größere Anzahl unterschiedlicher Daten aus den im Fahrzeug verbauten Sensoren und Steuergeräten, als es bei OBD möglich ist. Dazu zählen beispielsweise Daten zu Fahrzeugposition, Fahrzeugdynamik und Fahrzeugumgebung, Fahrzeugdiagnosedaten, Daten über die im Fahrzeug verbaute Hardware und deren Status, und Daten über den Antriebsstrang wie Ressourcenkonsum und genutzte Hardware samt Status.

Mit der zunehmenden Automatisierung von Fahrzeugen wird noch mehr leistungsfähige Sensorik, wie beispielsweise Radar oder Lidar verbaut. Damit erfassen diese Fahrzeuge noch mehr Daten über sich selbst und ihre Umgebung, und das in einer noch höheren Genauigkeit, was weitere innovative Anwendungsfälle möglich macht. Auch in diesem Zusammenhang wird sich zeigen, ob und wie Fahrzeugnutzer solche Systeme verwenden werden und ob sie genügend Vertrauen in solche Systeme setzen (Neuhuber et al. 2020). Neben der ökologischen Nachhaltigkeit durch ein „einprogrammiertes“, energieschonendes Fahren in elektrifizierten Fahrzeugen sollen diese Systeme signifikant dazu beitragen, Unfallzahlen weiter zu reduzieren und die Sicherheit im Straßenverkehr zu erhöhen.

Abhängig von Verkehrsdichte, befahrenem Straßentyp und Fahrzeugsegment ergeben sich laut einer Studie des Fraunhofer-Instituts für System- und Innovationsforschung (ISI) durch die Automatisierung von Fahrzeugen Potenziale zur signifikanten Reduktion des Kraftstoffverbrauchs im Maximalfall von bis zu $42 \%$. Gemittelt über die Anteile der Fahrten auf den unterschiedlichen Straßentypen kann die Automatisierung und Vernetzung den Kraftstoffverbrauch am einzelnen Fahrzeug immerhin noch um bis zu 17\% senken (Krail et al. 2019). Ein zukünftig weit verbreiteter Einsatz hochautomatisierter Fahrzeuge könnte Treibhausgasemissionen entweder massiv erhöhen oder drastisch reduzieren, was zum großen Teil von der öffentlichen Politik abhängen wird (Greenwald und Kornhauser 2019).

In Bezug auf zukünftige Geschäftsmodelle durch die automatisierte Mobilität wird sich zeigen, ob unbemannte hochautomatisierte Fahrzeuge, sogenannte Robotertaxis, in einen nachhaltigen Betrieb genommen werden können. Auch wird die Frage diskutiert werden müssen, ob hochautomatisierte Fahrzeuge den Verkehr im urbanen Raum weiter anwachsen lassen, da solche Fahrzeuge nicht auf einen festen Parkplatz am Wohnsitz des Besitzers angewiesen sind, sondern im automatisierten, unbemannten Modus einen entfernten Parkplatz ansteuern bzw. bei Bedarf durch den Besitzer von diesem Parkplatz gerufen werden können. Auch in Bezug auf hochautomatisierte Fahrzeuge wird sich ein maximaler Effekt auf die ökologische Nachhaltigkeit wohl nur dann ergeben, wenn diese Fahrzeuge geteilt genutzt werden, bzw. wenn sich Personen zusammenfinden, welche ein solches Fahrzeug gemeinsam und gleichzeitig für eine Fahrstrecke verwenden und damit auf ein privates Fahrzeug überhaupt verzichten. 


\section{Zusammenfassung und Ausblick}

Nach einer kurzen Einführung in das Konzept der Nachhaltigkeit und dem Beitrag digitaler Technologien als Befähiger für nachhaltige Mobilität sowie der Perspektive der Wirtschaftsinformatik auf dieses Thema wird mit Hilfe dreier Fallbeispiele veranschaulicht, wie der Einsatz digitaler Technologien im Kontext der Fahrzeugnutzung einen konkreten Beitrag zur (ökologischen) Nachhaltigkeit liefern kann.

Die Zukunft wird eine höhere Automatisierung von Fahrzeugen bringen, welche schrittweise über die zunehmende Verbreitung und Nutzung immer leistungsfähigerer Assistenzsysteme erfolgen wird. Auch die Verkehrsinfrastruktur wird mit mehr und mehr Sensoren versehen werden, um ihrerseits Daten über den Verkehr sowie über Umweltbedingungen zu sammeln und mit den Fahrzeugen zu kommunizieren. Eine Kommunikation zwischen Fahrzeugen und Verkehrsinfrastruktur soll neue Arten von Assistenzsystemen möglich machen. Das kann positive Auswirkungen auf die ökologische Nachhaltigkeit haben, wie die ersten beiden Fallbeispiele gezeigt haben. Ferner bleibt spannend zu beobachten, welche nachhaltigen Mobilitätsdienstleistungen sich zukünftig am Markt positionieren können und ob diese in der Lage sind, breite Bevölkerungsschichten zu überzeugen, die Anzahl privater Kraftfahrzeuge nachhaltig zu reduzieren, wie in Fallbeispiel drei diskutiert wird.

Fest steht bereits heute, dass die von Fahrzeugen generierten Daten einen wesentlichen Bestandteil zukünftiger Dienste darstellen werden und dass das Angebot an solchen Daten für Dritte durch die Automatisierung von Fahrzeugen und die Verfügbarkeit von Datenmarktplätzen und Datenportalen weiter zunehmen wird. Fahrzeugdaten ermöglichen vor allem in der Kombination mit anderen Daten wie beispielsweise Wetterdaten oder Daten über Fahrende eine Vielzahl an neuartigen Anwendungen. Viele Anwendungsmöglichkeiten sind heute noch der Fiktion zuzuordnen. In diesem Zusammenhang empfehlen Kaiser et al. (2018) die Prägung des Begriffs, Vehicle Information Systems, als Hinweis auf eine eigene Klasse von Informationssystemen und thematisieren eine zukünftige Rolle der Wirtschaftsinformatik im Hinblick auf die Entwicklung und Evaluierung solcher Systeme. Wie bei Informationssystemen üblich, lassen sich Effekte nur dann erzielen, wenn diese auch genutzt werden. Die Wirksamkeit von Informationssystemen hängt von deren Funktionalität und Nutzungsintensität ab, welche wiederum von Nutzerzufriedenheit und Benutzbarkeit beeinflusst wird, ein Phänomen, dass in der Wirtschaftsinformatik bereits intensiv untersucht wurde. Auch automatisierte Fahrzeuge und die damit möglich werdenden Geschäftsmodelle können nur dann einen maximalen Effekt auf die ökologische Nachhaltigkeit erzielen, wenn sie als Bestandteil von Mobilitätsdienstleistungen durch die breite Masse gemeinsam genutzt werden.

Danksagung The EVOLVE project (www.evolve-h2020.eu) has received funding from the European Union's Horizon 2020 research and innovation program under grant agreement No 825061. The document reflects only the author's views and the Commission is not responsible for any use thatmay be made of information contained therein. 


\section{Literatur}

Baptista P, Melo S, Rolim C (2014) Energy, environmental and mobility impacts of car-sharing systems. Empirical results from Lisbon, Portugal. Procedia 111:28-37

Bardhi F, Eckhardt GM (2012) Access-based consumption: the case of car sharing. J Consumer Res 39(4):881-898

Barkenbus JN (2010) Eco-driving: an overlooked climate change initiative. Energy Policy 38(2):762-769

Birrell SA, Fowkes M, Jennings PA (2014) Effect of using an in-vehicle smart driving aid on real-world driver performance. IEEE Trans Intell Transp Syst 15(4):1801-1810. https://doi.org/10.1109/TITS. 2014.2328357

Blohm I, Leimeister JM (2013) Gamification-design of IT-based enhancing services for motivational support and behavioral change. Bus Inf Syst Eng 5(4):275-278

Bolderdijk JW, Knockaert J, Steg EM, Verhoef ET (2011) Effects of pay-as-you-drive vehicle insurance on young drivers' speed choice: results of a Dutch field experiment. Accid Anal Prev 43(3):1181-1186

Bozem K, Nagl A, Rennhak C (2013) Energie für nachhaltige Mobilität. Springer, Wiesbaden

Brand KW, Jochum G (2000) Der deutsche Diskurs zu nachhaltiger Entwicklung. MPS-Texte. Münchner Projektgruppe für Sozialforschung e. V., (Jan 1, 2000)

vom Brocke J, Watson RT, Dwyer C, Elliot S, Melville N (2013) Green information systems: directives for the IS discipline. CAIS 33(1):30

Busse S, Hanelt A, Kolbe L (2014) Nachhaltige Mobilität - Herausforderungen und Potentiale für die Wirtschaftsinformatik. In: Brenner W, Hess T (Hrsg) Wirtschaftsinformatik in Wissenschaft und Praxis. Springer Gabler, Berlin, Heidelberg, S 141-152

de Camargo Fiorini P, Jabbour CJ (2017) Information systems and sustainable supply chain management towards a more sustainable society: where we are and where we are going. Int J Inf Manag 37(4):241-249

Chaloupka C, Kölbl R, Loibl W, Molitor R, Nentwich M, Peer S, Risser R, Sammer G, Schützhofer B, Seibt C (2015) Nachhaltige Mobilität aus sozioökonomischer Perspektive - Diskussionspapier der Arbeitsgruppe „Sozioökonomische Aspekte“ der ÖAW-Kommission „Nachhaltige Mobilität https:// doi.org/10.1553/ITA-ms-15-02

Clausen U, Klinger M (2018) Automatisiertes Fahren. In: Neugebauer R (Hrsg) Digitalisierung. Springer Vieweg, Berlin, Heidelberg, S 385-411

Degirmenci K, Katolla TM, Breitner MH (2015) How can mobile applications reduce energy consumption? An experimental investigation of electric vehicles. In: ECIS 2015 completed research papers (Paper 36)

Eissen M, Metzger JO, Schmidt E, Schneidewind U (2002) 10 years after Rio-concepts on the contribution of chemistry to a sustainable development. Angew Chem Int Ed 41(3):414-436

Elkington J (1997) The triple bottom line. In: Environmental management: readings and cases, SAGE Publications, 2. Aufl.

Elkington J, Rowlands IH (1999) Cannibals with forks: the triple bottom line of 21st century business. Altern J 25(4):42

Firnkorn J, Müller M (2011) What will be the environmental effects of new free-floating car-sharing systems? The case of car2go in Ulm. Ecol Econ 70(8):1519-1528

Furuhata M, Dessouky M, Ordóñez F, Brunet ME, Wang X, Koenig S (2013) Ridesharing: the state-ofthe-art and future directions. Transp Res Part B 57:28-46

Geissdoerfer M, Savaget P, Bocken NM, Hultink EJ (2017) The circular economy-A new sustainability paradigm? J Clean Prod 143:757-768

Gholami R, Sulaiman AB, Ramayah T, Molla A (2013) Senior managers' perception on green information systems (IS) adoption and environmental performance: results from a field survey. Inf Manag 50(7):431-438

Greenwald JM, Kornhauser A (2019) It's up to us: policies to improve climate outcomes from automated vehicles. Energy Policy 127:445-451

Hamari J, Koivisto J, Sarsa H (2014) Does gamification work? A literature review of empirical studies on gamification. In: 47th Hawaii international conference on system sciences 6. Jan. 2014, S 3025-3034

Herrenkind B, Harnischmacher C, Willnat M, Lembcke TB, Villbrandt Y (2020) Digital shared mobility services-A literature analysis and avenues for IS-related future research. In: AMCIS 2020 Proceedings

Herwig N (2017) Sharing Economy - Neue Geschäftsmodelle der urbanen Mobilität. IZNE Working Paper Series 
Kaiser C, Stocker A, Fellmann M (2019) Understanding Data-driven Service Ecosystems in the Automotive Domain. In: Americas Conference on Information Systems

Kaiser C, Stocker A, Festl A, Lechner G, Fellmann M (2018) A research agenda for vehicle information systems. In: European Conference on Information Systems

Kaiser C, Stocker A, Festl A, Djokic-Petrovic M, Papatheocharous E, Wallberg A, Ezquerro G, Ortigosa Orbe J, Szilagyi T (2020) A vehicle telematics service for driving style detection: implementation and privacy challenges. In: 6th International Conference on Vehicle Technology and Intelligent Transport Systems VEHITS 2020, Prague, Czech Republic, May 2-4, 2020, S 29-36

Kollosche I, Schwedes O (2016) Mobilität im Wandel: Transformationen und Entwicklungen im Personenverkehr. https://library.fes.de/pdf-files/wiso/12702.pdf. Zugegriffen: 28. Juli 2020

Kosch B, Wagner H (2010) Alles im grünen Bereich - Mit Green IT zu Energieeffizienz und Nachhaltigkeit. In: Spath D, Bauer W, Rief S (Hrsg) Green Office. Gabler, Wiesbaden, S 205-212

Krail M, Hellekes J, Schneider U, Dütschke E, Schellert M, Rüdiger D, Steindl A, Luchman I, Waßmuth V, Flärnig H, Schade W, Mader M (2019) Energie- und Treibhausgaswirkungen des automatisierten und vernetzten Fahrens im Straßenverkehr, Wissenschaftliche Beratung des BMVI zur Mobilitäts- und Kraftstoffstrategie. www.isi.fraunhofer.de/content/dam/isi/dokumente/ccn/2019/energietreibhausgaswirkungen-vernetztes-fahren.pdf. Zugegriffen: 28. Juli 2020

Kumar S, Teichman S, Timpernagel T (2012) A green supply chain is a requirement for profitability. Int J Prod Res 50(5):1278-1296

Lehner M, Mont O, Heiskanen E (2016) Nudging-A promising tool for sustainable consumption behaviour? J Clean Prod 134:166-177

Loose W (2014) Vom Solidarhandeln zur Marktorientierung. In: Bundesverband CarSharing e. V (Hrsg.) 25 Jahre Carsharing im Überblick. Verkehrszeichen 30 (2). KSV-Verlag, S. 17-18

Magana VC, Munoz-Organero M (2015) GAFU: using a gamification tool to save fuel. IEEE Intell Transp Syst Mag 7(2):58-70

McIlroy RC, Stanton NA (2015) A decision ladder analysis of eco-driving: the first step towards fuel-efficient driving behaviour. Ergonomics 58(6):866-882. https://doi.org/10.1080/00140139.2014.997807

Melville NP (2010) Information systems innovation for environmental sustainability. MIS Q 34(1):1-21

Messner D, Nakicenovic N, Zimm C, Clarke G, Rockström J, Aguiar AP, Boza-Kiss B, Campagnolo L, Chabay I, Collste D, Comolli L (2019) The digital revolution and sustainable development: opportunities and challenges (Report prepared by The World in 2050 initiative). http://pure.iiasa.ac.at/id/ eprint/15913. Zugegriffen: 28. Juli 2020

Neuhuber N, Lechner G, Kalayci TE, Stocker A, Kubicek B (2020) Age-related differences in the interaction with advanced driver assistance systems-A field study. In: International Conference on HumanComputer Interaction 19. Juli 2020 Springer, Cham, S 363-378

Nijland H, van Meerkerk J (2017) Mobility and environmental impacts of car sharing in the Netherlands. Environ Innov Soc Transit 23:84-91

Ott K, Döring R (2011) Theorie und Praxis starker Nachhaltigkeit. Metropolis, Marburg

Pagoropoulos A, Pigosso DC, McAloone TC (2017) The emergent role of digital technologies in the circular economy: a review. Procedia CIRP 64:19-24

Reich A, Krämer NA, Lenninger R (2018) Fahrzeugdaten-Management Standardisierter Zugang als Basis für neue Geschäftsmodelle. ATZelektronik 13(2):42-47

Ruhrort L (2019) Ausgangslage und Problemaufriss: Nachhaltige Mobilität als gesellschaftlicher Transformationsprozess. In: Transformation im Verkehr. Springer VS, Wiesbaden, S 23-47

Stocker A, Kaiser C, Fellmann M (2017) Quantified vehicles. Novel services for vehicle lifecycle. Data Bus Inf Syst Eng 59(2):125-130

Tils G, Rehaag R, Glatz A (2015) Carsharing - ein Beitrag zu nachhaltiger Mobilität. Working Papers des KVFNRW,, Bd. 2. Verbraucherzentrale Nordrhein-Westfalen e. V.; Kompetenzzentrum Verbraucherforschung NRW, Düsseldorf https://doi.org/10.15501/kvfwp_2

VDA (2017a) Zugang zum Fahrzeug und zu im Fahrzeug generierten Daten. www.vda.de/de/services/ Publikationen/zugang-zum-fahrzeug-und-zu-im-fahrzeug-generierten-daten.html. Zugegriffen: 28. Juli 2020

VDA (2017b) Data security for networked mobility. How "NEVADA-Share \& Secure" works. www.vda. de/en/topics/innovation-and-technology/data-security/how-it-works.html. Zugegriffen: 28. Juli 2020

Vereinte Nationen (2015) Transforming our world: the 2030 agenda for sustainable development. Division for Sustainable Development Goals, New York

Watson RT, Boudreau MC, Chen AJ (2010) Information systems and environmentally sustainable development: energy Informatics and new directions for the IS community. MIS Quarterly, Vol. 34, No. 1 (March 2010), S 23-38. https://doi.org/10.2307/20721413 
Weber M, Gsell M, Fanderl N, Krauß J, Kern M (2020) Mobilität 4.0 - Digitale Plattformen als Beitrag zur nachhaltigen Verkehrswende in Stadt und Land? Kurzpapier zur Analyse mobilitätsspezifischer digitaler Plattformsysteme. www.oeko.de/fileadmin/oekodoc/regGEM-digital-Mobilitaet.pdf. Zugegriffen: 28. Juli 2020

Weinmann M, Schneider C, vom Brocke J (2016) Digital nudging. Bus Inf Syst Eng 58(6):433-436

Zimmermann S, Angerer P, Provin D, Nault BR (2018) Pricing in C2C sharing platforms. J Assoc Inf Syst 19(8):4 